\title{
Valproate causes metabolic disturbance in normal
}

\section{man}

\author{
DM TURNBULL,* DJ DICK,* LESLEY WILSON, $\ddagger$ HSA SHERRATT, $\dagger$ \\ KGMM ALBERTI
}

From the Departments of Neurology (Muscular Dystrophy Research Laboratories),, Clinical Biochemistry and Metabolic Medicine $\ddagger$ and Pharmacological Sciences, $\dagger$ University of Newcastle upon Tyne, Newcastle upon Tyne, UK

SUMMARY Valproate is an important anticonvulsant which is rarely associated with fatal hepatotoxicity. Previous experiments have shown that valproate inhibits several metabolic processes in isolated rat hepatocytes and when administered to starved rats causes a fall in the blood concentrations of glucose and ketone bodies. Since these changes may be related to the hepatotoxicity, the effect of valproate administration on intermediary metabolism in man was studied. One gram of valproate given orally to fasted normal humans caused a $78 \%$ fall in the concentration of 3-hydroxybutyrate and a $60 \%$ fall in total ketones. Also the concentrations of lactate, pyruvate, alanine and glycerol increased after valproate administration. Similar changes were observed after intravenous administration of $400 \mathrm{mg}$ of valproate. Valproate clearly has a significant effect on intermediary metabolism in the liver and this is probably related to the mechanism of the hepatotoxicity.

Valproate administered as the sodium salt or as valproic acid, is a widely used and effective anticonvulsant. Whilst serious adverse effects are uncommon when valproate is administered as monotherapy, ${ }^{1}$ associated fatal hepatotoxicity has been described. Hepatic failure is acute and has some similarities with Reye's syndrome. ${ }^{2}$ Valproate (di-npropylacetate) is a branched-chain fatty acid which inhibits fatty acid and pyruvate oxidation, fatty acid and urea synthesis, and gluconeogenesis in isolated rat hepatocytes. ${ }^{34}$ Administration of valproate to starved rats resulted in a fall in blood glucose and ketone body concentrations, ${ }^{3}$ consistent with the inhibition of gluconeogenesis and of fatty acid oxidation observed in isolated rat hepatocytes. Valproate is also hypoglycaemic and hypoketonaemic in diabetic ketoacidotic rats. ${ }^{5}$

The only previous studies on the effect of valproate on intermediary metabolism in humans were those of Mortensen et al ${ }^{6}$ who found that patients on valproate therapy excreted dicarboxylic acids suggesting impaired $\beta$-oxidation of fatty acids, and of

Address for reprint requests: Dr DM Turnbull, Muscular Dystrophy Group Research Laboratories, Newcastle General Hospital, Newcastle upon Tyne NE46BE, UK.

Received 26 February 1985 and in revised form 20 July 1985. Accepted 23 July 1985

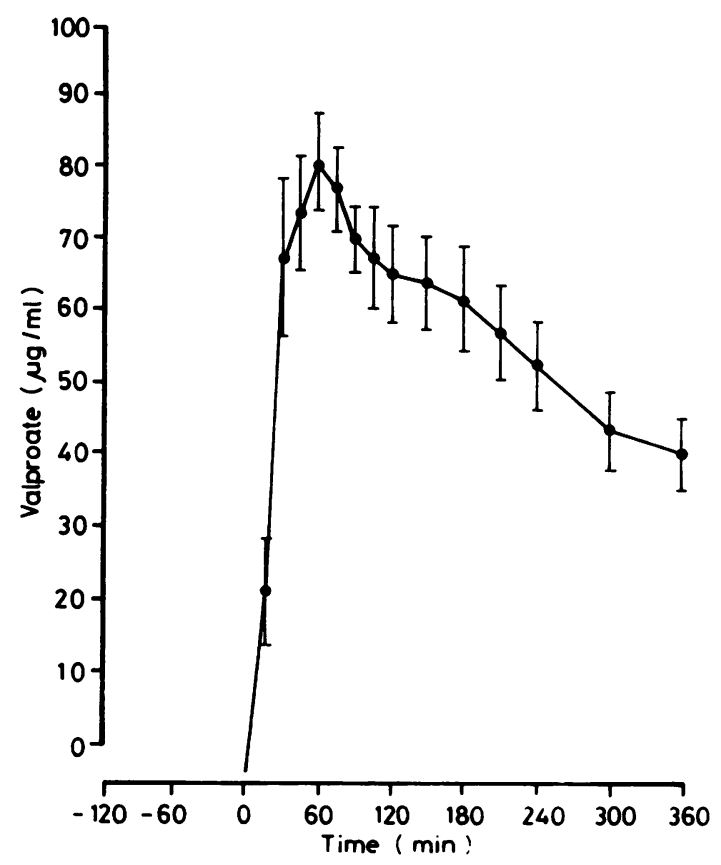

Fig 1 Plasma valproate concentration after oral administration $(1 \mathrm{~g})$. Results are mean $\pm S E M$ for five subjects. 

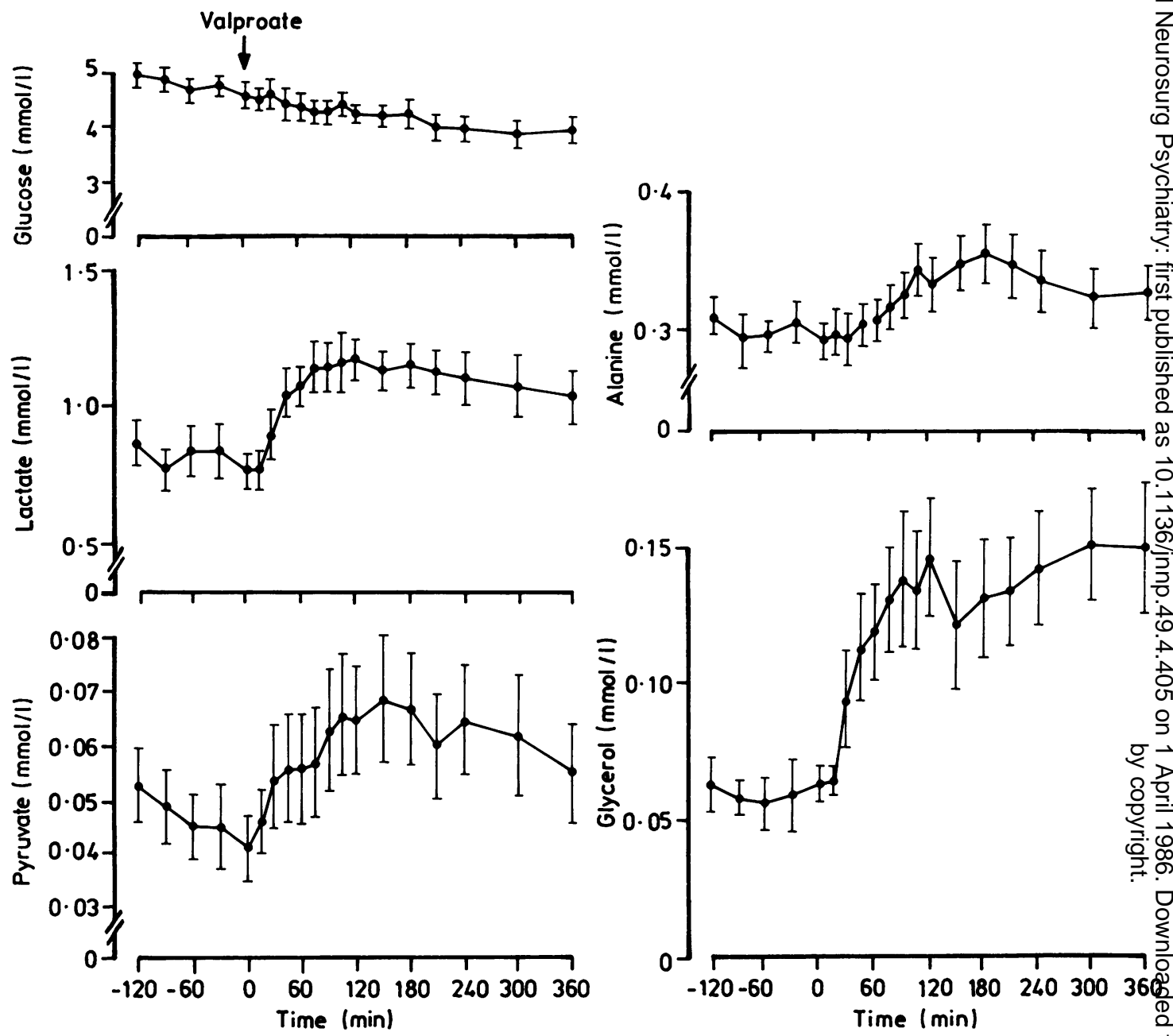

Fig 2 Effect of oral administration of valproate ( $1 \mathrm{~g}$ ) on blood metabolite concentrations. Results are mean $\pm S E M$ for five subjects.

Thurston et $^{7} \mathrm{l}^{7}$ who found decreased blood concentrations of 3-hydroxybutyrate in children on valproate therapy. This may suggest a mechanism for the hepatotoxicity if similar changes in intermediary metabolism to those observed in rats occur in humans. We have therefore determined the effects of valproate in normal man.

\section{Methods}

Five healthy male subjects within $10 \%$ of ideal body weight, were fasted for 36 hours before intravenous administration of valproate, and for 18 hours prior to oral administration. An indwelling intravenous cannula was inserted at 07.30 hours and patency maintained by flushing with $0 \cdot 154 \mathrm{M} / 1$ saline. Five basal samples were taken between 08.00 and 10.00 hours and sodium valproate was then administered either as $400 \mathrm{mg}$ intravenously or as $1.0 \mathrm{gm}$ orally (nonenteric coated tablets). Blood samples were then taken every 15 minutes for 2 hours and subsequently at intervals of 0.5-1.0 hour until 6 hours (oral) or 5.5 hours (intravenous) (see figures). The subjects were static during this time. The study was approved by the Newcastle University Ethical 윽 Committee and all subjects gave informed oral consent.

Blood glucose, 3-hydroxybutyrate, lactate, pyruvate, alanine and glycerol concentrations were determined by an automated enzymatic fluorimetric method ${ }^{8}$ and blood ace- $\sigma$ toacetate concentration by a manual spectrophotometric $N$ method. ${ }^{9}$ Plasma concentrations of non-esterified fatty acids (NEFA) were measured by a radiocobalt technique. ${ }^{10} \mathrm{\omega}$ Serum insulin concentrations were measured by radio- 0 

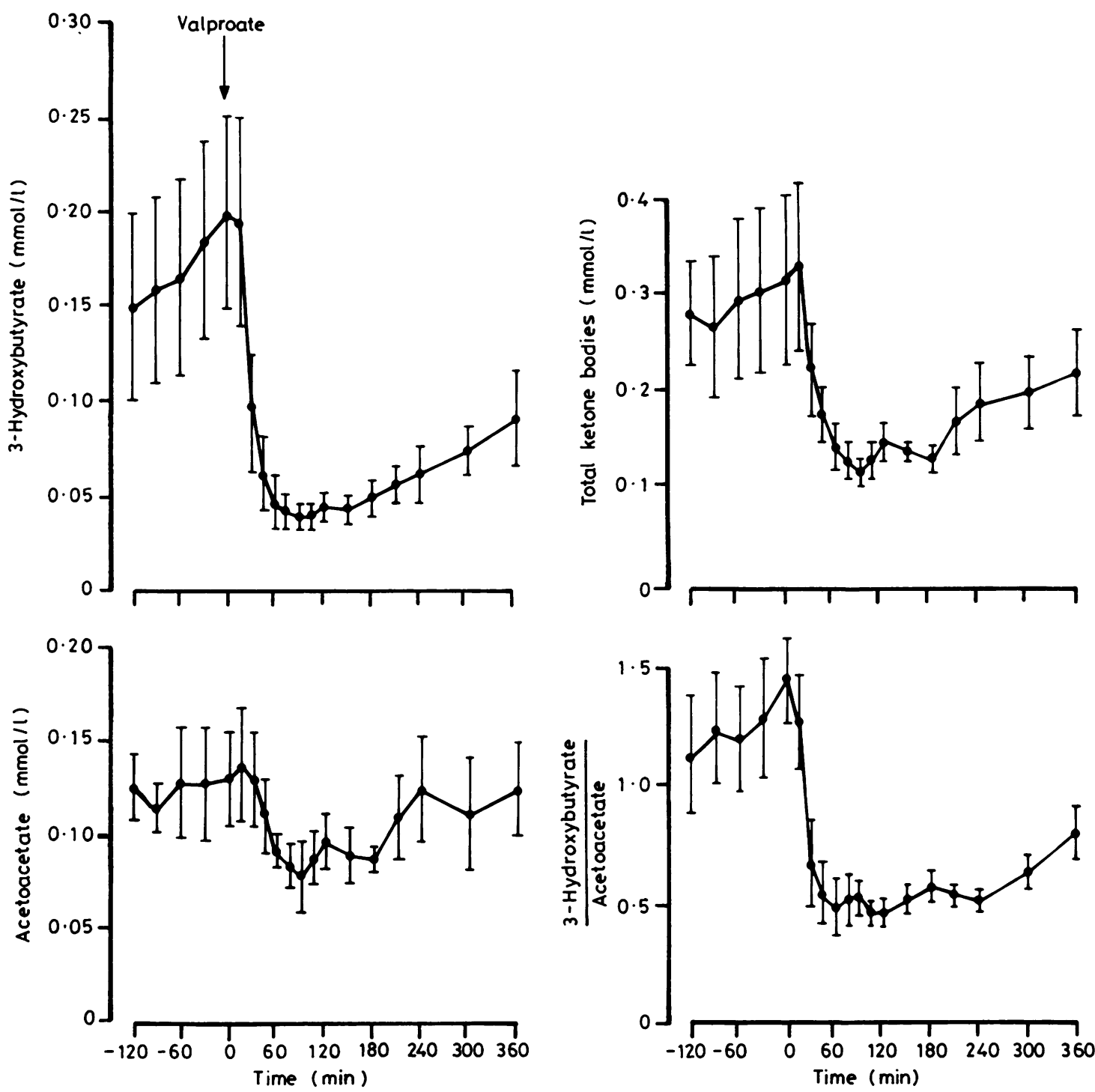

Fig 3 Effect of oral administration of valproate $(1 \mathrm{~g})$ on blood metabolite concentrations. Results are mean $\pm S E M$ for five subjects.

immunoassay. ${ }^{11}$ Plasma valproate concentrations were determined by gas chromatography. ${ }^{12}$

\section{Results}

\section{Oral administration}

The mean peak serum valproate concentration, after oral administration of $1.0 \mathrm{~g}$ of sodium valproate, was $80 \mu \mathrm{g} / \mathrm{ml}$ which is within the suggested therapeutic range of $50-100 \mu \mathrm{g} / \mathrm{ml}^{13}$ (fig 1). Blood glucose concentration fell by $17 \%$ over the 8 hours of the study, and the rate of fall did not appear to be affected by valproate administration (fig 2). Concentrations of lactate, pyruvate and alanine rose initially and then declined (fig 2). The concentration of glycerol was markedly increased by administration of valproate. Serum insulin remained at basal concentrations throughout the study. 

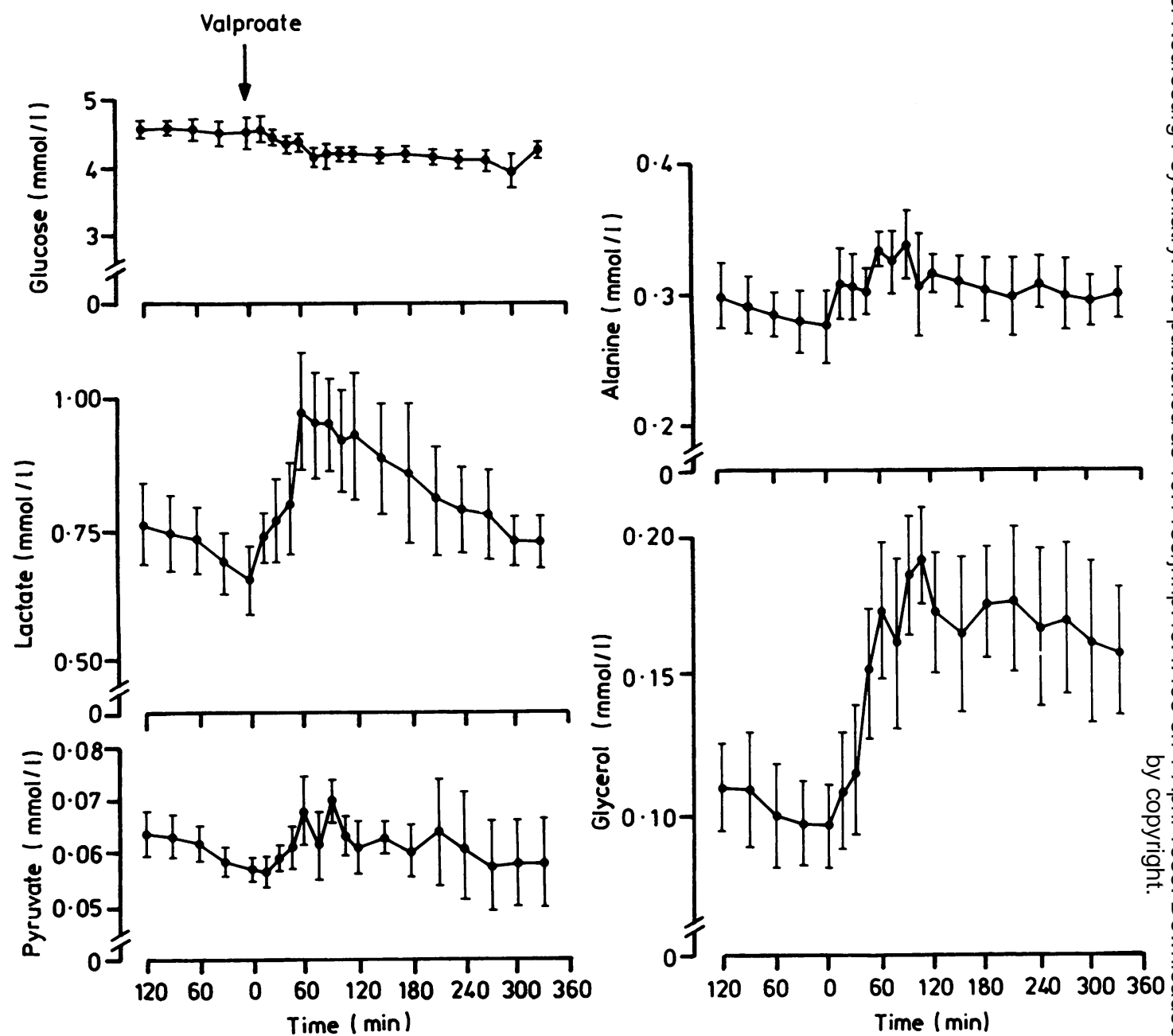

Fig 4 Effect of intravenous administration of valproate $(400 \mathrm{mg})$ on blood metabolite concentrations. Results are mean $\pm S E M$ for five subjects.

The most marked effect of valproate administration was on the blood concentration of ketone bodies (fig 3). There was a $78 \%$ fall in the concentration of 3-hydroxybutyrate, a $38 \%$ fall in acetoacetate concentration and a $60 \%$ fall in total ketone body concentrations. There was also a $65 \%$ fall in the 3-hydroxybutyrate/acetoacetate ratio. These effects on blood ketones were greatest 90 minutes after the administration of valproate but were still evident 6 hours later.

\section{Intravenous administration}

The subjects were fasted for longer ( 36 hours) prior to the intravenous administration of valproate $(400 \mathrm{mg})$. The effect on blood metabolite concentrations were similar to the effects of $1.0 \mathrm{gm}$ given orally, with a slight fall in blood glucose, and increases in lactate, pyruvate, alanine and glycerol (fig 4). The ketone body concentrations were again most affected, with a $\dot{0}$ $75 \%$ decrease in the concentration of 3 . 3-hydroxybutyrate, $40 \%$ decrease in acetoacetate concentration and the 3-hydroxybutyrate/ $₹$ acetoacetate ratio decreased by $63 \%$ (fig 5 ).

\section{Discussion}

Hepatotoxicity is the most serious adverse effect of valproate therapy. It is extremely rare and in the cases $\mathcal{N}$ reported death was due to fulminant hepatic failure $N$ or a Reye-like syndrome. Many of the patients $N$ affected are children with severe epilepsy, who were or 

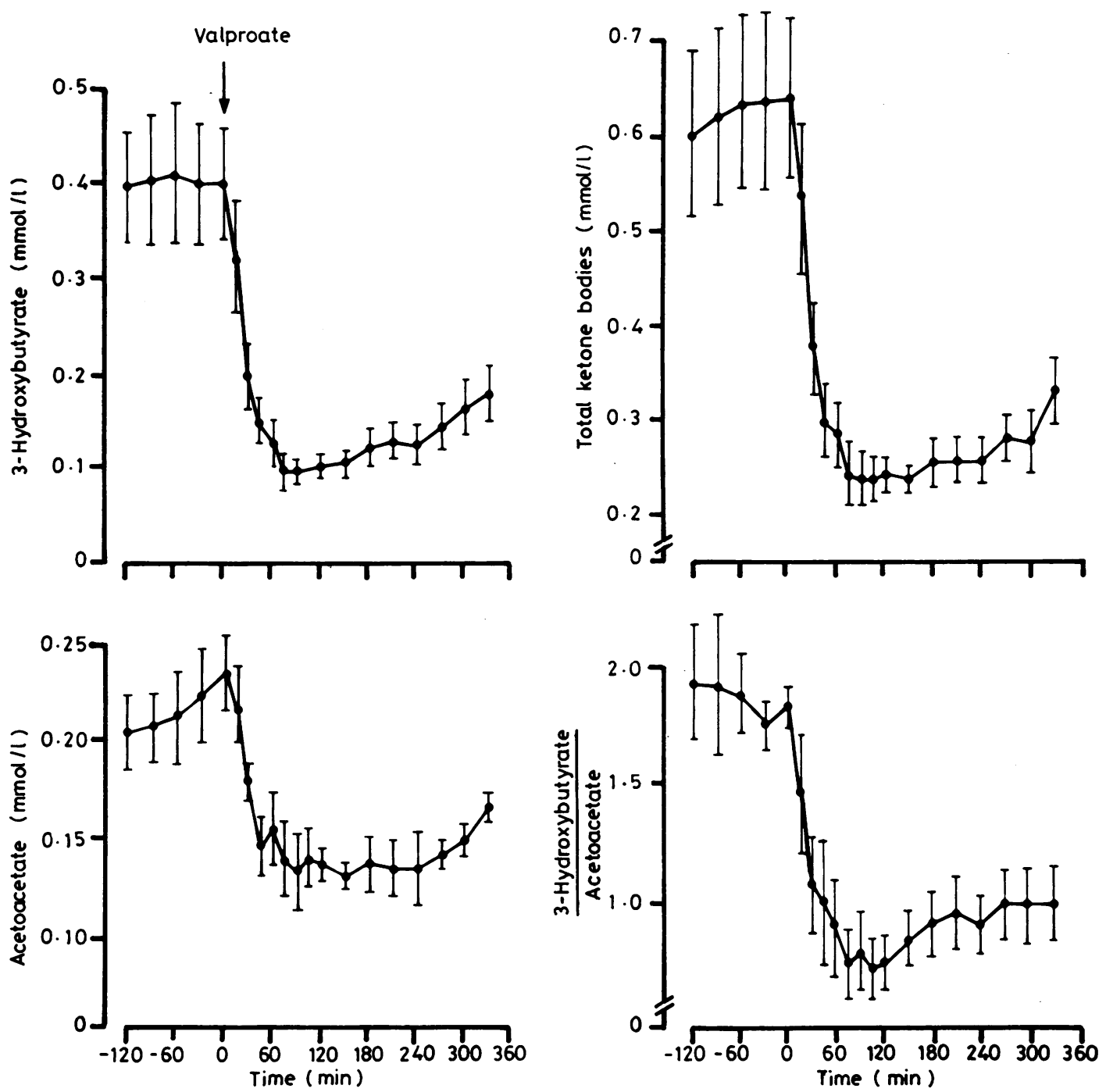

Fig 5 Effect of intravenous administration of valproate $(400 \mathrm{mg})$ on blood metabolite concentrations. Results are mean \pm SEM for five subjects.

had been taking other anticonvulsants in addition to valproate. ${ }^{14}$

Concentrations of valproate found in man during therapy inhibit fatty acid oxidation, fatty acid synthesis, pyruvate oxidation, urea synthesis and gluconeogenesis in isolated rat hepatocytes. ${ }^{34}$ All the metabolic disturbances caused by valproate can be explained by the increased acyl-CoA/CoASH ratio within the mitochondrial matrix due to the formation of valproyl-CoA by acyl-CoA synthetase in this compartment. $^{3}$ These results prompted this investigation into the effect of valproate on intermediary metabolism in humans. Administration of valproate to nor- mal fasted humans caused a marked lowering of blood ketone body concentrations. Ketone bodies are the major products of hepatic $\beta$-oxidation. Whilst falls in ketone body concentration may be partly due to increased utilisation in peripheral tissues, it is most likely that this indicates inhibition of fatty acid oxidation by valproate in human liver. Our results agree with those recently published by Thurston et al. ${ }^{7}$ These authors studied the effect of chronic valproate administration on blood metabolite concentrations in epileptic children and found a $67 \%$ decrease in the concentration of 3-hydroxybutyrate after an overnight fast. 
The metabolic response to fasting in control subjects has been extensively studied using the same laboratory techniques. ${ }^{15}$ The changes in metabolite concentrations in blood during fasting are gradual and not associated with marked fluctuations as seen after valproate administration. Johnston et al ${ }^{15}$ found that blood glucose concentrations fell by $18 \%$ and alanine concentrations by $40 \%$ over a period of fasting from 12 to 60 hours although the concentrations of blood lactate and pyruvate did not change. Blood glycerol and ketone bodies and the plasma concentration of NEFA rose steadily. The increased concentrations of lactate, pyruvate and alanine following administration of valproate suggests that there is partial inhibition of gluconeogenesis or of pyruvate oxidation or both. Changes in NEFA concentrations could not be measured accurately as valproate reacted variably with the radiocobalt assay. However, the results indicated qualitatively that the NEFA concentrations increased further following administration of valproate. These and the increases in glycerol concentrations suggest that lipolysis is increased.

Valproate clearly inhibits several metabolic pathways in humans. The partial inhibition of gluconeogenesis and of ketogenesis caused by valproate may impair liver function significantly and cause the toxicity when there is already an inborn error of metabolism or if the patients are on multiple drug therapy. Such a putative relationship with inborn errors may account for the increased incidence of valproate-induced toxicity in children. However, since these inhibitions are only partial it must be assumed that the majority of patients tolerate valproate without any adverse effects. Further, the metabolic effects of valproate are analogous to those seen in some inborn errors of metabolism, for example, isovaleric acidaemia and propionicacidaemia.

This investigation was supported by the Medical Research Council (DMT), by a Competitive Award for Research into Toxicological Mechanisms from the Wellcome Trust (HSAS, LW \& KGMMA), by Abbott Laboratories, Chicago, USA (DMT \& HSAS), and by the Muscular Dystrophy Group of Great Britain (DJD).

\section{References}

${ }^{1}$ Jeavons PM. Valproic acid: toxicity. In: Woodbury DM, Penry JK, Pippenger CE, eds. Antiepileptic Drugs. New York: Raven Press, 1982:601-10.

${ }^{2}$ Turnbull DM. Adverse effects of valproate. Adverse Drug Reactions and Acute Poisoning Review 1983;2:191-216.

${ }^{3}$ Turnbull DM, Bone AJ, Bartlett K, Koundakjian PP, Sherratt HSA. The effects of valproate on intermediary metabolism in isolated rat hepatocytes and intact rats. Biochem Pharmacol 1983;32:1887-92.

${ }^{4}$ Becker C-M, Harris RA. Influence of valproic acid on hepatic carbohydrate and lipid metabolism. Arch Biochem Biophys 1983;223:381-92.

${ }^{5}$ Turnbull DM, Bone AJ, Wilson L, Sherratt HSA. Hypoglycaemic and hypoketonaemic effects of valproate in the spontaneously diabetic B,B rat. Diabetes Res 1985:in press.

${ }^{6}$ Mortensen PB, Gregersen N, Kolvraa S, Christensen E. The occurrence of $\mathrm{C} 6-\mathrm{Cl} 0$ - dicarboxylic acids in urine from patients and rats treated with dipropylacetate. Biochem Med 1980;24:153-61.

${ }^{7}$ Thurston JH, Carroll JE, Dodson WE, Hauhart RE Tasch V. Chronic valproate administration reduces fasting ketonaemia in children. Neurology (Cleveland) 1983;33:1348-50.

${ }^{8}$ Lloyd B, Burrin J, Smyth P, Alberti KGMM. Simple automated enzymatic fluorimetric assays for blood glucose, lactate, pyruvate, alanine, glycerol and 3-hydroxybutyrate. Clin Chem 1978;24:1724-9.

${ }^{9}$ Williamson DH, Mellanby JH, Krebs HA. Enzymic deter mination of $\mathrm{D}(-)$-hydroxybutyrate and acetoacetate in blood. Biochem J 1962;82:90-6.

${ }^{10} \mathrm{Ho}$ RJ, Meng HC. A simple and ultrasensitive method for determination of free fatty acid by radiochemical assay. Anal Biochem 1969;31:426-36.

${ }^{11}$ Soeldner J, Slone D. Critical variables in the radioimmunoassay of serum insulin using the double antibody technic. Diabetes 1965;14:987-8.

${ }^{12}$ Chard CR. A simple method for the determination of Epilim in serum. In: Legg NJ, ed. Clinical and Pharmacological Aspects of Sodium Valproate (Epilim) in the treatment of epilepsy. Tunbridge Wells: MCS Consultants, 1976:89-91.

${ }^{13}$ Schobben F, van der Kleijn E. Valproate: biotransformation. In: Woodbury DM, Penry JK, Pippenger CE, eds. Antiepileptic Drugs New York: Raven Press, 1982;567-78.

${ }^{14}$ Editorial. Sodium valproate and the liver. Lancet 1980;2:1119-20.

${ }^{15}$ Johnston DG, Blesa-Malpica G, Burrin JM, et al. Dopamine blockade inhibits starvation ketosis in man. Clin Endocrinol 1983;19:389-96. 